\title{
EMBALAGENS DE MEDICAMENTOS GENÉRICOS E RISCO DE ERRO DE MEDICAÇÃO: UM ESTUDO COM PROFISSIONAIS DE FARMÁCIAS
}

\author{
Carla Patrícia de Araújo Pereira \\ Universidade Federal de Campina Grande \\ carlapereira@usp.br \\ Jádira Soares de Farias \\ Universidade Federal de Campina Grande \\ jadirasoares@gmail.com \\ Addizza de Carvalho Ganem \\ Universidade Federal de Campina Grande \\ addizzagn@hotmail.com
}

Resumo: Embalagens de medicamentos têm um papel importante na transmissão de informações essenciais aos usuários. Na literatura, são reportados casos em que drogas foram trocadas por profissionais dentro de centros médicos, devido a informações confusas ou à semelhança das embalagens. Este artigo apresenta resultados iniciais de pesquisa que investiga a influência do design na compreensão de informações em embalagens de medicamentos genéricos. Relata um estudo no qual foram realizadas entrevistas com farmacêuticos e balconistas de farmácias. 0 levantamento buscou identificar dificuldades enfrentadas em suas rotinas de trabalho, causadas pela semelhança entre embalagens ou outros aspectos do design, e conhecer os mecanismos adotados para prevenção de erros. Este estudo constatou que o sistema atual de padronização das embalagens de medicamentos genéricos no Brasil dificulta o trabalho dos profissionais das farmácias e pode induzir a erro. Os problemas descritos dizem respeito à dificuldade de distinção entre os fármacos, mas também à identificação de concentração, quantidade e prazo de validade.

Palavras-chave: embalagens de medicamentos, erros de medicação, design de informação.

\begin{abstract}
Packaging represent an important role on communicate essential information to users of medicines. In literature, are reported cases in which drugs were mistaken by practitioners within medical centers due to confusing information or packaging similarity. This article presents partial results of research that investigates the influence of design on understanding information in packaging of generic medicines. Reports a study in which interviews were carried out with pharmacists and pharmacy clerks. The survey sought to identify difficulties faced on their work routines, caused by packaging similarities or other aspects of design, in
\end{abstract}


addition to acknowledge the mechanisms adopted to prevent errors. This study has noted that the current packaging standardization system of generic medicines in Brazil hinders the work of professionals in pharmacies and can induce to error. The issues reported are related to the difficulty to distinguish drugs, but also to the identification of concentration, amount of drug and expiration date.

Keywords: packaging of medicines, medication errors, information design.

\section{INTRODUÇÃO}

No Brasil, é produzida e comercializada uma grande variedade de medicamentos que cresce a cada dia. Por razões diversas, profissionais de saúde e pessoas comuns lidam diariamente com uma quantidade considerável dessas substâncias potencialmente perigosas, as quais, se não forem administradas adequadamente, trazem riscos à saúde. As embalagens de medicamentos têm um papel importante na transmissão de informações essenciais aos usuários e são manipuladas por diferentes grupos de pessoas, abrangendo desde médicos, enfermeiros e farmacêuticos, aos próprios pacientes em tratamento.

Erros de medicação são um problema de saúde pública que atinge as diferentes populações em âmbito mundial. Na literatura especializada, são reportados diversos eventos em que medicamentos trocados por profissionais dentro de centros médicos colocam em risco a vida de pacientes. Na Inglaterra, estima-se que um terço dos erros de medicação registrados pela National Health Service (NHS) sejam causados pela má interpretação de embalagens e rótulos (WILKINSON, 2009).

Entre os diversos casos reportados na literatura, Cohen (2007) relata um número significativo de erros ocorridos devido à má interpretação da informação de quantidade associada à concentração do produto: na situação específica, embalagens contendo $20 \mathrm{ml}$ de um antibiótico na concentração de $40 \mathrm{mg} / \mathrm{ml}$ foram confundidas pelos usuários, que interpretaram que o frasco inteiro continha apenas $40 \mathrm{mg}$ da substância, causando uma superdosagem massiva.

Diferentes fatores influenciam a interpretação das informações contidas em embalagens de medicamentos, como o tipo e tamanho da fonte tipográfica, as cores e contrastes utilizados no design, o formato dos recipientes, assim como outros elementos relacionados à rotulagem, embalagem e nomenclatura. Nudka e Leff (2001) descrevem uma situação na qual uma ampola de água foi confundida com uma substância anestésica devido à semelhança entre as embalagens. Exemplos com este apontam para o perigo potencial decorrente da falta de um sistema de organização e diferenciação das embalagens de medicamentos, o que pode induzir a erros de medicação, seja em hospitais, em farmácias, ou nas residências dos pacientes.

A semelhança entre embalagens de medicamentos diferentes é frequentemente apontada como fator de risco. Muitas empresas tendem a adotar o mesmo design de embalagem para a maior parte dos medicamentos que produzem, o que dificulta a distinção entre as diferentes substâncias e pode trazer consequências graves. Cohen (2007) relata a repetida administração de um medicamento broncodilatador a pacientes que deveriam receber vitamina $E$, ocorrida no Canadá. No caso, as embalagens dos produtos, produzidos pela mesma indústria farmacêutica, 
eram praticamente indistinguíveis uma da outra: embalagens de vidro de $15 \mathrm{ml}$ com o mesmo layout e cores no rótulo.

Profissionais que trabalham com o manejo de medicamentos diariamente confirmam que distinguir os medicamentos entre si pode ser uma tarefa árdua quando as embalagens são muito semelhantes. Lalor (2011) aponta que a função principal de uma embalagem é assegurar que o medicamento possa ser facilmente identificável pelos profissionais de saúde e pelo consumidor. Segundo o autor, a troca entre produtos que possuem nomes, embalagens e rótulos similares é tida como a maior causa de erro por profissionais da área de saúde ao suprir um paciente. O erro pode acontecer em qualquer fase do manejo do medicamento e traz um risco significativo para a segurança dos pacientes, seja em hospitais e farmácias ou na própria comunidade.

No Brasil, um levantamento recente realizado na farmácia de um hospital identificou semelhanças relativas a rótulos de medicamentos como causas potenciais para erros de dispensação, armazenamento e administração em hospitais (LOPES et al., 2012). Em relação às embalagens de medicamentos destinados à venda aos pacientes em farmácias, não há dados disponíveis.

Este artigo apresenta resultados iniciais de pesquisa em curso que investiga a influência do design na compreensão de informações em embalagens de medicamentos, com foco no problema da identificação correta dos fármacos. Descreve uma investigação realizada com embalagens de medicamentos genéricos, na qual se buscou identificar os recursos visuais utilizados para destacar as informações mais relevantes. Relata um estudo feito com um grupo de farmacêuticos e atendentes de farmácias, com o objetivo de conhecer o papel desempenhado pelo design na tarefa de repassar o medicamento correto aos pacientes no ato da compra.

O caráter essencialmente empírico dos dados apresentados deve-se ao fato de se tratar de um relato de etapa inicial de pesquisa. Trata-se de estudo de caráter exploratório, cujos resultados ainda serão submetidos a análises mais aprofundadas, com base em referencial teórico específico, visando à formulação de hipóteses a serem investigadas em etapas posteriores.

\section{EMBALAGENS DE MEDICAMENTOS GENÉRICOS}

No Brasil, os medicamentos são categorizados em três tipos: os de referência, os medicamentos similares e os genéricos. Nos três casos, de acordo com o risco que podem representar aos pacientes, podem ser adquiridos sem prescrição ou com prescrição médica. A Lei dos Medicamentos Genéricos (Lei no 9.787, de 1999) faz parte de uma política implantada pelo Governo Federal com o objetivo de estimular a concorrência e a variedade de oferta de remédios, melhorar a qualidade dos medicamentos, reduzir os preços e facilitar o acesso da população aos tratamentos.

Segundo a Agência Nacional de Vigilância Sanitária (ANVISA), "o medicamento genérico é aquele que contém o mesmo princípio ativo, na mesma dose e forma farmacêutica, é administrado pela mesma via e com a mesma posologia e indicação terapêutica do medicamento de referência" (ANVISA, 2015). Este tipo de medicamento não possui nome fantasia (marca própria), portanto em sua embalagem é possível identificar somente o princípio ativo do medicamento e a marca do fabricante.

A ANVISA é o órgão responsável pela normatização das embalagens de medicamentos. Os genéricos devem estar em conformidade com a Resolução RDC no 
71 - que estabelece regras para a rotulagem de medicamentos em geral - e também com a RDC $n \circ 47$ - referente à diferenciação da rotulagem dos medicamentos genéricos e dos demais. Pelas normas em vigor, o medicamento genérico deve ser identificado por uma faixa amarela contendo a letra " $G$ ", onde também deve constar a inscrição "medicamento genérico" (RDC 47/2001; RDC 71/2009). Além da faixa amarela específica para identificação dos genéricos, as embalagens de medicamentos com venda sob prescrição médica devem possuir uma faixa vermelha ou preta, conforme o tipo de substância sujeita ou não a controle especial (RDC 71/2009).

Cada indústria farmacêutica deve seguir as normas, porém, nos espaços permitidos, podem utilizar o seu próprio padrão gráfico (cores, fontes tipográficas, diagramação, etc.), diferenciando suas embalagens dos demais fabricantes. Observa-se que o padrão gráfico de cada marca se repete em todas as embalagens de medicamentos genéricos produzidos pelo mesmo fabricante, o que gera a seguinte situação: enquanto medicamentos iguais são comercializados em embalagens graficamente diferentes (se produzidos por empresas diferentes), medicamentos distintos são disponibilizados à população em embalagens iguais (se fabricados pelo mesmo laboratório), conforme demonstrado na Figura 1.

\section{MÉTODO}

O estudo, de caráter exploratório, foi realizado a partir de visitas a seis farmácias da cidade de Campina Grande, PB, no primeiro semestre de 2016. As lojas foram selecionadas por fazerem parte de redes de farmácias que, somadas, têm amplo alcance na cidade, sendo responsáveis por suprir grande parte da população. As visitas visaram observar as embalagens e as formas como são organizadas para venda. Foram consideradas na pesquisa apenas as embalagens de medicamentos genéricos vendidos sob prescrição médica. Na ocasião, foram realizadas entrevistas estruturadas com funcionários responsáveis pelo repasse dos medicamentos aos usuários durante a compra. Para cada farmácia, foi entrevistado o farmacêutico responsável e um balconista, totalizando 12 informantes.

As entrevistas visaram identificar possíveis dificuldades enfrentadas em suas rotinas de trabalho causadas pela semelhança entre as embalagens de medicamentos ou outros aspectos do design, e conhecer possíveis mecanismos adotados para prevenção de erros na identificação das substâncias. As entrevistas foram apoiadas por formulários previamente elaborados, preenchidos pelas pesquisadoras, contendo um total de 15 questões, entre perguntas fechadas e abertas. Antes das entrevistas, cada participante assinou o respectivo Termo de Consentimento Livre e Esclarecido (TCLE), conforme determinação do Comitê de Ética em Pesquisa com Seres Humanos da Universidade Federal de Campina Grande (CEP/UFCG).

\section{RESULTADOS E DISCUSSÃO}

Durante as entrevistas, os farmacêuticos foram solicitados a estimar a quantidade de medicamentos genéricos à venda em suas respectivas farmácias. A exceção de dois entrevistados que não realizaram a estimativa, os demais informaram que lidam com um número superior a 50 medicamentos genéricos comercializados sob prescrição médica, podendo chegar a um total superior a 500 medicamentos, variando de acordo com o tamanho e estoque das farmácias visitadas. 
Em relação à quantidade de laboratórios que suprem as farmácias, metade dos entrevistados informou que trabalha com até 10 empresas diferentes e um terço deles lida com um número superior a 20 fabricantes. Metade das farmácias comercializa até 30 tipos de medicamentos diferentes por fabricante, e algumas delas podem trabalhar com 100 tipos diferentes ou mais por fabricante. Embora estes números resultem de estimativas feitas pelos próprios trabalhadores e não por uma contagem de estoque, eles evidenciam a grande quantidade e variedade de produtos com os quais estes profissionais lidam diariamente, sendo todos medicamentos que requerem prescrição médica, portanto, sustâncias potencialmente perigosas que exigem uma identificação precisa ao serem repassados aos usuários.

\subsection{0 problema da semelhança e estratégias para prevenção de erros}

$O$ atual sistema de padronização das embalagens de medicamentos genéricos no Brasil prioriza a distinção visual das embalagens exclusivamente em relação à particularização dos fabricantes. As cores predominantes das embalagens auxiliam apenas na identificação das empresas e a semelhança das embalagens de todos os medicamentos de um mesmo fabricante é um problema real para os profissionais das farmácias, conforme relatos fornecidos.

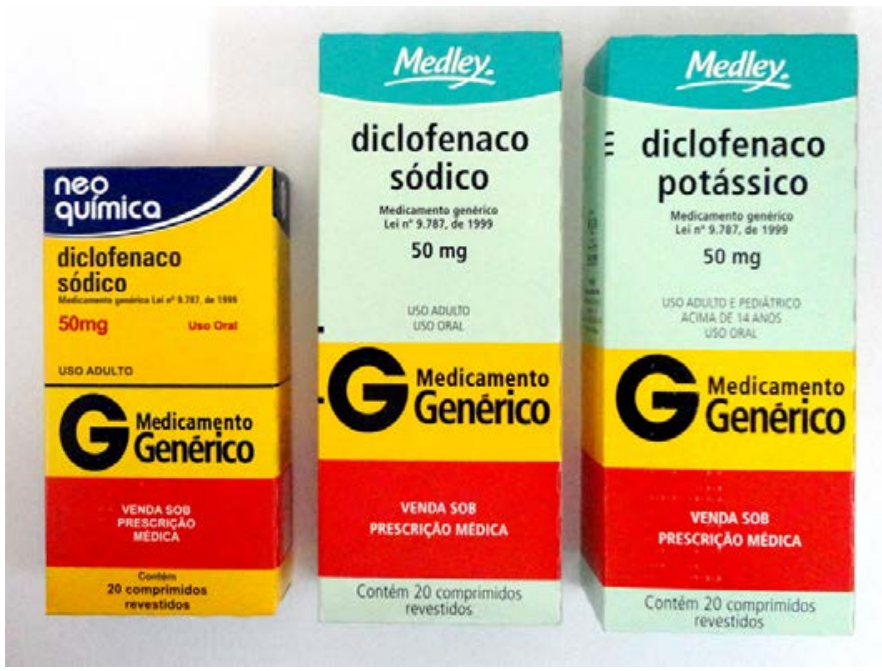

Figura 1 - Medicamentos iguais comercializados em embalagens graficamente diferentes e medicamentos distintos em embalagens iguais.

Fonte: Elaborado pelas autoras.

Entre os casos referidos nas entrevistas, verifica-se a semelhança na embalagem entre medicamentos com grande potencial de risco aos pacientes em tratamento. A figura 01 apresenta um conjunto de anti-inflamatórios. Na imagem, observa-se que o diclofenaco sódico e o diclofenaco potássico produzidos pelo mesmo fabricante possuem embalagens visualmente indistintas. Ambos os medicamentos apresentam a mesma concentração $(50 \mathrm{mg})$, o mesmo tamanho e formato de embalagem, as mesmas cores, a mesma quantidade de comprimidos por caixa e pertencem à mesma classe terapêutica. Contudo, de acordo com os entrevistados, o diclofenaco sódico é contraindicado para indivíduos que sofrem de hipertensão arterial, uma doença muito comum no Brasil. A única diferença perceptível entre os 
medicamentos é o nome do princípio ativo, que neste caso, possui grafia e sonoridade semelhantes, o que agrava o problema.

Os procedimentos adotados nas farmácias para evitar a troca de medicamentos durante a venda são: a conferência da medicação por duas pessoas diferentes (balconista e caixa), conferência pelo sistema de cadastro dos medicamentos, a atenção do próprio atendente ao nome da substância e a forma de organização das embalagens na prateleira.

Em alguns casos, os medicamentos são separados por fabricante, mas na maioria das farmácias visitadas eles são organizados pelo nome dos fármacos dispostos em ordem alfabética (Figura 2). Também na maioria dos casos, a forma de organização é um sistema padrão adotado não apenas pela loja visitada, mas por todas as farmácias da mesma rede. A maioria dos profissionais entrevistados (farmacêuticos e atendentes) considerou eficiente a forma de organização empregada em suas respectivas lojas. Eles relataram que a disposição das embalagens de medicamentos pelo nome da substância em ordem alfabética torna a procura mais rápida e eficiente. Informaram ainda que este sistema auxilia também no treinamento dos atendentes recém-contratados.

Contudo, apesar da tarefa de localização dos medicamentos nas prateleiras basear-se na leitura do nome da substância (princípio ativo do medicamento), o conflito entre informação verbal e visual pode induzir a erro. Cientes desta possibilidade, os próprios profissionais das farmácias adotam sistemas adicionais de sinalização para minimizar o risco. Durante as visitas, foi observado o uso de etiquetas inseridas nas embalagens a fim de prevenir erro na identificação dos medicamentos (Figura 2).

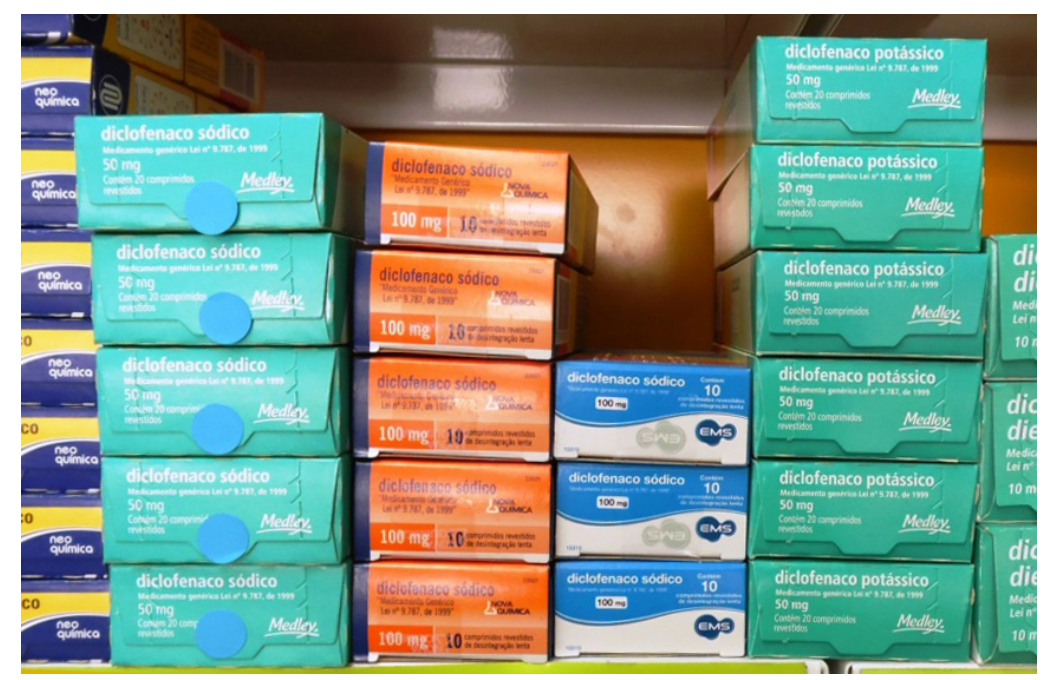

Figura 2 - Organização dos medicamentos em ordem alfabética e uso de etiquetas para diferenciar os fármacos devido à semelhança nas embalagens.

Fonte: Elaborado pelas autoras.

$\mathrm{Na}$ Figura 2 observam-se medicamentos dispostos na prateleira numa organização em ordem alfabética. As etiquetas circulares nas embalagens do diclofenaco sódico foram inseridas pelos funcionários para evitar a troca com o diclofenaco potássico do mesmo fabricante. Os medicamentos em questão estão empilhados e separados em colunas distintas, mas esta forma de disposição não é uma 
regra. Noutra situação registrada, ambos os fármacos estão empilhados numa mesma coluna (Figura 3).

Observa-se ainda que nem sempre as embalagens são dispostas nas prateleiras com o painel principal voltado para frente. Em muitos casos, é a face superior da embalagem que fica visível, na qual o espaço disponível para as informação é menor, o nome da substância é escrito numa linha única, o que diminui substancialmente o tamanho da fonte tipográfica (Figura 3).

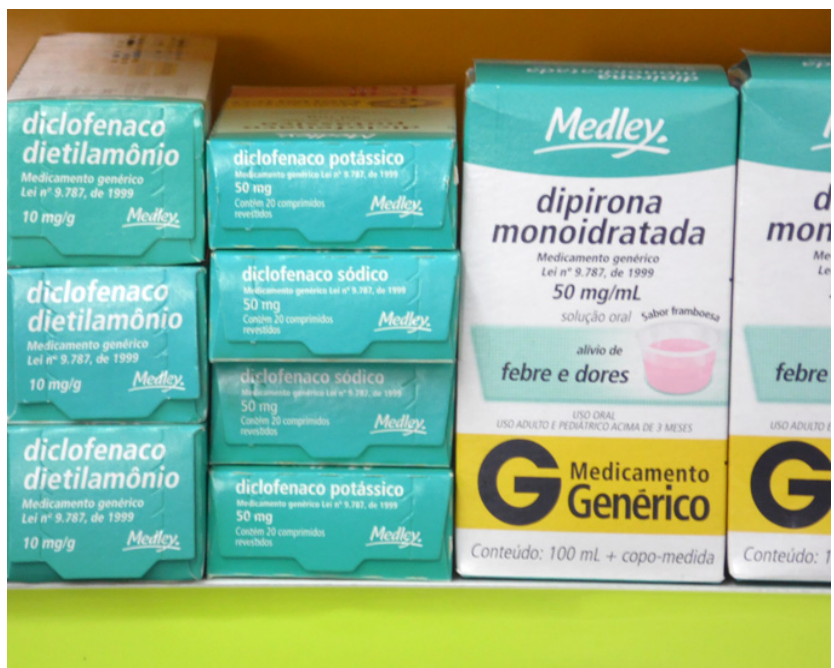

Figura 3 - Fármacos diferentes em embalagens iguais empilhados numa única coluna.

Fonte: Elaborado pelas autoras.

O uso de adesivos em embalagens graficamente iguais também foi observado para assinalar diferenças na concentração do fármaco e na quantidade de comprimidos. Em certos casos, etiquetas são fixadas para destacar medicamentos cuja data de validade está próxima, sendo esta informação (data de validade) frequentemente mencionada como de difícil localização. Este uso de marcadores inseridos nas embalagens pelos profissionais das farmácias demonstra a ineficiência do sistema gráfico atual das embalagens de medicamentos genéricos, que não segue preceitos básicos do design de informação no que se refere à hierarquização e destaque das informações relevantes.

\subsection{Elementos de destaque}

Os entrevistados também foram perguntados sobre os aspectos das embalagens que mais se destacam quando são observadas. Como pode ser visto na Tabela 1, a maioria deles referiu-se ao nome do medicamento (citado por 58,3\% dos informantes), seguido da cor (mencionada por $41,7 \%)$, nome do fabricante (25\%) e dosagem (16,7\%).

Nas embalagens observadas, em geral o excesso de informação visual, as faixas coloridas obrigatórias e a marca do fabricante competem visualmente com o nome da substância (Figura 4). Desse modo, a atenção dos profissionais voltada à leitura do nome do fármaco, única forma possível de identificação no atual sistema das embalagens de medicamentos, parece ser a explicação mais provável para este elemento ter sido considerado o de mais destaque pela maioria dos informantes. 
Em relação às cores, consideradas pelos entrevistados uma das características que mais chama a atenção, elas não contribuem para a identificação dos fármacos. Nas embalagens de medicamentos genéricos vendidos sob prescrição médica, elas têm um papel informativo em relação a três outros aspectos: a indicação da necessidade de apresentação ou retenção de receita médica (faixa vermelha ou preta), a diferenciação do genérico dos medicamentos similares ou de referência (faixa amarela), e a identificação do fabricante (cor predominante do design).

Tabela 1 - Questão 10: ‘Quando você observa uma embalagem de medicamento, quais aspectos se destacam mais?'

\begin{tabular}{lll}
\hline Aspectos que se destacam na embalagem & № & $\%$ \\
\hline Nome do medicamento & 7 & 58,3 \\
\hline Cor & 5 & 41,7 \\
\hline Nome do fabricante & 3 & 25,0 \\
\hline Dosagem & 2 & 16,7 \\
\hline Formato & 0 & 0 \\
\hline Outros & 1 & 8,3 \\
\hline Total de respondentes & $12^{*}$ & $100,0^{*}$ \\
\hline
\end{tabular}

*Alguns entrevistados indicaram mais de uma resposta (Total de entrevistados $=12$ )

Fonte: Elaborado pelas autoras, com base na pesquisa realizada.

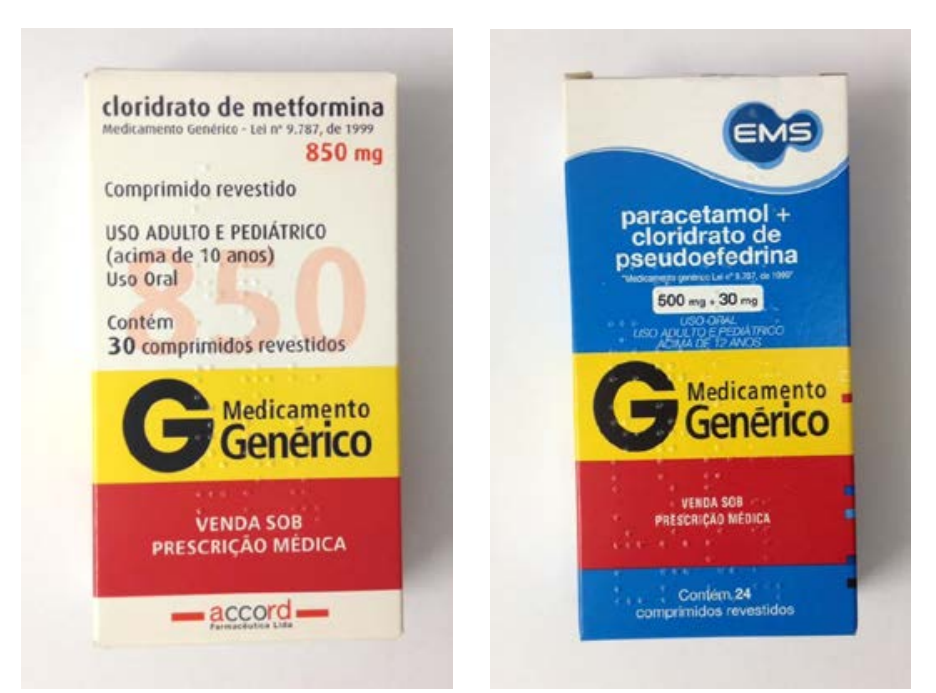

Figura 4 - Excesso de informação, faixas coloridas e marca do fabricante concorrem visualmente com o nome do fármaco.

Fonte: Elaborado pelas autoras.

Para identificar corretamente o medicamento no momento de repassá-lo aos clientes, os farmacêuticos e atendentes declararam observar principalmente o nome da substância e dosagem (ambos citados por 75\% dos profissionais entrevistados), mas também o nome do fabricante $(33,3 \%)$ e a cor $(16,7 \%)$ da embalagem, conforme exposto na Tabela 02 .

$\mathrm{O}$ atual sistema de padronização das embalagens de medicamentos genéricos gera problemas reais na identificação dos medicamentos: a maioria dos entrevistados $(66,6 \%)$ declarou que os atendentes têm dificuldade para identificar corretamente os produtos cujas embalagens são semelhantes. Apesar dos procedimentos de segurança 
adotados - sistema de organização em ordem alfabética, atenção voltada à leitura do nome da substância e uso eventual de etiquetas - $75 \%$ dos profissionais entrevistados declararam que já houve casos de erro no repasse do medicamento ao cliente por causa da semelhança da embalagem (Tabela 3). Para os pacientes que precisam adquirir medicamentos para fazer seu tratamento em casa, o risco de erro de medicação se inicia na própria farmácia: $100 \%$ dos entrevistados informaram que algum cliente já levou um medicamento errado para casa.

Tabela 2 - Questão 11: ‘No momento de repassar o produto para o cliente, quais aspectos da embalagem vocês observam para identificar corretamente o medicamento?’

\begin{tabular}{lll}
\hline Aspectos observados na identificação do medicamento & № & $\%$ \\
\hline Nome do medicamento & 9 & 75,0 \\
\hline Dosagem & 9 & 75,0 \\
\hline Nome do fabricante & 4 & 33,3 \\
\hline Cor & 2 & 16,7 \\
\hline Formato & 0 & 0 \\
\hline Outros & 4 & 33,3 \\
\hline Total de respondentes & $12^{*}$ & $100,0^{*}$ \\
\hline
\end{tabular}

*Alguns entrevistados indicaram mais de uma resposta (Total de entrevistados $=12$ )

Fonte: Elaborado pelas autoras, com base na pesquisa realizada.

Tabela 3 - Questão 12: 'Já houve casos de erro no repasse do medicamento ao cliente por causa da semelhança da embalagem?

\begin{tabular}{lll}
\hline Ocorrência de erro devido à semelhança da embalagem & No & $\%$ \\
\hline Sim & 9 & 75,0 \\
\hline Não & 3 & 25,0 \\
\hline Total de respondentes & 12 & 100,0 \\
\hline
\end{tabular}

Fonte: Elaborado pelas autoras, com base na pesquisa realizada.

Além da questão da identificação correta do fármaco, os profissionais das farmácias também mencionaram dificuldades para diferenciar a concentração e quantidade de comprimidos devido à semelhança do design, além de dificuldade na localização/leitura do prazo de validade. O repasse incorreto de medicamentos que possuem o mesmo princípio ativo, mas que apresentam concentração e/ou quantidade distinta pode induzir a superdosagem ou subdosagem, o que também representa risco à saúde dos pacientes.

\section{CONCLUSÃO}

Este estudo constatou que o sistema atual de padronização das embalagens de medicamentos genéricos no Brasil, que prioriza a distinção visual das embalagens exclusivamente em relação à particularização dos fabricantes, dificulta o trabalho dos profissionais das farmácias. A legislação vigente focaliza a diferenciação entre 0 medicamento genérico e os similares ou de referência, mas é omissa no que se refere à distinção entre os fármacos.

Embora os farmacêuticos e atendentes sejam conscientes da importância da leitura do nome do princípio ativo nas embalagens, e trabalhem com um sistema de organização em ordem alfabética nas prateleiras visando à prevenção de erros, o 
conflito entre informação verbal e informação visual torna a tarefa de identificação mais lenta e difícil, sobretudo diante da grande quantidade de medicamentos com os quais trabalham diariamente. Os problemas relatados dizem respeito à distinção entre os fármacos, mas também à identificação de concentração, quantidade e prazo de validade.

A comercialização de medicamentos iguais em embalagens graficamente diferentes em conjunto com a disponibilização de medicamentos distintos em embalagens iguais é, por princípio, um sistema que induz a erro. Esta pesquisa constatou que se trata de um problema real enfrentado pelos profissionais de farmácias e, para os usuários de medicamentos genéricos, o risco de utilizar o medicamento de forma incorreta origina-se na própria farmácia, durante a compra.

Recomenda-se a realização de estudos complementares que abordem o modo como os usuários (profissionais e pacientes) percebem e interpretam as informações nas embalagens de medicamentos, uma área do design de informação ainda carente de estudos no Brasil, bem como a formulação de propostas de intervenção no sistema gráfico atual das embalagens, visando à prevenção de erros de medicação.

\section{REFERÊNCIAS}

BRASIL. Ministério da Saúde. Agência Nacional de Vigilância Sanitária. Resolução RDC № 47, 28 mar. 2001. Diário Oficial, Brasília, DF, 30 mar. 2001. Seção 1, p. 57.

. Resolução RDC № 71, 22 dez. 2009. Diário Oficial, Brasília, DF, 23 dez. 2009. Seção 1, p. 75-80.

BRASIL. Poder Legislativo. Lei № 9.787, 10 fev.1999. Diário Oficial, Brasília, DF, 11 fev. 1999. Seção 1, p. 57-58.

BRASIL. ANVISA. Medicamentos genéricos. Disponível na internet por http em: <http://portal.anvisa.gov.br/wps/content/Anvisa+Portal/Anvisa/Inicio/Medicamentos/ Assunto+de+Interesse/Medicamentos+genericos/Medicamento+Generico>. Acesso em: 26 out. 2015.

COHEN, Michael R. The role of drug packaging and labeling in medication errors. In: COHEN, Michael R. (Ed.). Medication errors. 2nd ed. Washington: American Pharmacists Association, 2007. p. 111-152.

LALOR, Daniel. Medicines labelling. Australian Prescriber, v. 34, n. 5, oct. 2011. Disponível na internet por http em: <http://dx.doi.org/10.18773/austprescr. 2011.072>. Acesso em: 07 dez. 2015.

LOPES, Diana M. A. et al. Análise da rotulagem de medicamentos semelhantes: potenciais erros de medicação. Revista da Associação Médica Brasileira, v. 58, n. 1, p. 95-103, fev. 2012.

NDUKA, C.; LEFF, D. Mistaken identity. British Medication Journal, v. 323, p. 615, 15 sep. 2001. Disponível na internet por http em: <http://www.ncbi.nlm.nih.gov/pmc/ articles/PMC1121186/>. Acesso em: 15 out. 2015.

WILKINSON, Emma. Safety by design. Chemist \& Druggist, 2009, p. 30-31. Disponível na internet por http em: <http://www.chemistanddruggist.co.uk/content/rolepackaging-dispensing-errors >. Acesso em: 30 ago. 2015. 